TM-1663

[SSCL-N-720]

\title{
An Application of Differential Geometry to SSC Magnet End Winding"
}

\author{
J. M. Cook \\ Argonne National Laboratory \\ 9700 S. Cass Avenue \\ Argonne, Illinois 60439
}

April 1990 


\title{
An Application of Differential Geometry to SSC Magnet End Winding
}

\author{
J. M. Cook* \\ Advanced Photon Source \\ Argonne National Laboratory \\ Argonne, IL 60439
}

April 1990

* On loan to Fermi National Accelerator Laboratory, Batavia, IL 60510, operated by the Universities Research $A$ ssociation, Inc., under contracts with the U.S. Department of Energy. 


\section{Introduction}

It is expected that a large fraction of the total cost of the proposed Superconducting Supercollider will be spent on magnets, and, as Leon Lederman has remarked (on page 181 of reference [8]), "most of the cost of making a magnet is in the ends." Among the mechanical problems to be solved there is the construction of an end-configuration for the superconducting cables which will minimize their strain energy. The purpose of this paper is to promote the use of differential geometry in this minimization. The use will be illustrated by a specific application to the winding of dipole ends. The cables are assumed to be clamped so firmly that their strain is not altered by Lorentz stresses.

A reasonable mathematical model of the cables can be obtained from the well-known Euler-Kirchhoff theory of thin rods described, for example, in Chapters 18 and 19 of [10]. To find the equilibrium configuration of a cable according to this theory, a system of three coupled, first order, ordinary differential equations must be solved subject to given initial and final conditions and, in our case, subject also to the condition that the cable be constrained to lie against a cylindrical surface coaxial with the bore of the magnet. There is no provision for shearing or extensibility. The elastic properties of the rod are completely characterized by one torsional and two flexural coefficients of rigidity expressing the Bernoulli-Euler relations connecting stress-couples with curvature and twist (see equation (12) on page 388 of [10]).

Because an unstressed cable more resembles a long flat rectangle than it does a rod, one of its flexural rigidities is much larger than the other. It is much easier to bend the cable about a fold line in the rectangle than about a line perpendicular to the plane of the rectangle. Therefore, as a preliminary approximation to be relaxed later, we take one of the two flexural rigidities to be infinite. This is implied by what is called the "constant perimeter condition" by practitioners of the art of magnet winding [14]. Then the cable can be modeled purely geometrically as a two-dimensional strip to be bent into three-dimensional space so that the length of every arc in the strip is left unchanged. (By Maxwell's definition (page 81 of [11]) "The operation of bending is a continuous change of the form of a surface, without extension or contraction of any part of it", and the definition has become part of modern terminology [12].) The bent strip is part of a developable surface (see the bottom of page 303 in [4], or top of page 44 in volume $I$ of [15]). Straight lines in the unstrained (planar) strip become geodesics in the bent strip. Less obviously, any smooth curve in space uniquely determines, except possibly where its curvature is zero, a developable surface in which the 
curve is a geodesic. This surface is called the rectifying developable of the curve (see page 47 of [15]). In particular, the entire configuration of the bent strip is uniquely determined by that of (either) one of its edges. Instead of solving for the configuration of the strip in space, with an edge constrained to lie on the given cylindrical surface, we need only solve for that of a curve already in the surface. As in reference [14] we will call this curve the base curve of the strip. The system of three, coupled, ordinary differential equations is thereby reduced to one, second order, ordinary differential equation, unconstrained except by initial and final conditions. The method to be described in this paper will be based on the rectifying developable.

There is a deviation from adherence to the constant perimeter condition that this rectifying developable method, or any other method, must necessarily adapt to. As the cable comes down the long straight section of a (for example) dipole magnet it will lie along a straight line, a line of zero curvature. This is the singular situation mentioned above. The rectifying developable is not uniquely determined. Any plane containing the straight line will trivially be a developable surface containing it as a geodesic. The angle of inclination of the cable along the straight section of the dipole will select a particular one of these planes but, having been chosen for other reasons, it will not in general have the same angle of inclination as that of the rectifying developable containing the bent strip at the end section of the magnet where the two sections meet. The bent strip must be twisted to bring it into coincidence with the straight strip at this junction. The constant perimeter condition must be violated. No method can avoid this violation. Our aim will be to take into account the finiteness of the flexural rigidity temporarily assumed infinite, and describe a computationally feasible algorithm for determining the configuration of the strip by minimizing the total strain energy while satisfying the initial condition that it meet the straight section smoothly. The angle of twist of the strip away from the rectifying developable will be assumed small but the method, as implemented, and in skilled hands, seems also to work for large twists.

The minimization will be effected by an iterative procedure, a direct method (see Chapter IV, $\$ 2$ in [5]) from the calculus of variations designed to take advantage of the special structure of our particular problem. The procedure contains two steps:

Step 1: Minimize the strain energy by varying the base curve (within the cylindrical surface) while holding the other edge of the strip, the free edge, fixed relative to a certain family of coordinate systems attached to the base curve. 
Step 2: Minimize the strain energy by varying the free edge of the strip while holding the base curve fixed.

These two steps are repeated alternately. In the finite difference approximation described in this paper, with physically justifiable conditions on the strain energy functional, the functional will be approximable in a neighborhood of its minimum by a quadratic form. We construct an initial guess within this neighborhood by using the special geometric structure of our problem. Then the alternating-step iteration procedure, with suboptimization in each step in two orthogonal subspaces spanning the entire space of independent variables, will converge. An unconstrained optimization technique can be used because the independent variables will be chosen so that the constraints are automatically satisfied.

There is no iteration within Step 1, and the number of iterations within Step 2 can be abbreviated early in the procedure when complete convergence within Step 2 is not necessary. And because we have an adequate guess at the general shape of the optimal free edge, it can be reparameterized in such a way as to greatly decrease the number of independent variables whose values are adjusted in Step 2. This splitting of the set of independent variables into two disjoint subsets in Steps 1 and 2, and then the reduction of their number in Step 2, is done in a physically meaningful way facilitating interactive monitoring and even intervention into the convergence process.

Section 2 of this paper describes Step 1. Section 3 describes the rectifying developable. Section 4 describes the necessary twisting of the strip away from developability in Step 2. Section 5 describes the implementation of the algorithms in FORTRAN and the results of some experience with the method.

All of the theory of curves and surfaces that we will need was put into its final form by the 19 th century differential geometers. We will use that theory in an intuitive way, in the spirit of 19 th century geometry, without going into details of mathematical rigor which can be found in standard 20th century books on the subject. Reference [15], and Chapters III and VIII in [4] are good introductions.

\section{Base Curves}

Denote the base curve by the vector-valued function $\vec{R}(s)$ of its arc length measured from $s=0$ at the end of the straight section where the end winding begins. In the Euler- 
Kirchhoff theory the strain energy density along the strip, per unit of $s$, is given by

$$
\frac{1}{2}\left(a_{1} \kappa_{1}(s)^{2}+a_{2} \kappa_{2}(s)^{2}+a_{3} \tau(s)^{2}\right)
$$

where $a_{1}$ and $a_{2}$ are the flexural rigidities of the strip about axes perpendicular to $\vec{R}$ and tangent and perpendicular to the strip respectively (see page 269 in [7]), and $a_{3}$ is the torsional rigidity of the strip about $\vec{R}$ (see page 270 in [7]). $\kappa_{1}(s)$ and $\kappa_{2}(s)$ are the components of curvature of $\vec{R}$ at $s$ perpendicular and tangent to the strip respectively, and $\tau(s)$ is the twist of the strip about $\vec{R}$ at $s$.

The curvature $\kappa(s)$ of $\vec{R}$ at $s$ equals $\sqrt{\kappa_{1}(s)^{2}+\kappa_{2}(s)^{2}}$. Define $\varphi$ by $\cos \varphi(s)=$ $\kappa_{1}(s) / \kappa(s)$ with the sign of $\varphi(s)$ left indeterminate until Section 4 after more geometry has been set up. Then the strain energy density becomes

$$
\frac{1}{2}\left(a_{1} \kappa(s)^{2} \cos ^{2} \varphi(s)+a_{2} \kappa_{2}(s)^{2}+a_{3} \tau(s)^{2}\right) .
$$

It will be seen in Section 4 that $\varphi(s)$ is the angle of twist of the strip at $s$ away from the rectifying developable. By the constant perimeter condition, $\kappa_{2}(s)$ must be kept small for all $s$ so the method assumes that $\varphi$ is small and that $\cos ^{2} \varphi \approx 1$ (it equals 1 to within second order in $\varphi$ ) and that the energy density can be adequately approximated by

$$
\frac{1}{2}\left(a_{1} \kappa(s)^{2}+a_{2} \kappa_{2}(s)^{2}+a_{3} \tau(s)^{2}\right) .
$$

( $a_{2}$ is large so the second term should not be neglected.)

By equations (5) and (6) on page 284 of [4],

$$
\begin{aligned}
& \gamma=\kappa \sin \Phi, \\
& k=\kappa \cos \Phi,
\end{aligned}
$$

where $\gamma$ and $k$ are the geodesic and normal curvatures of the base curve in the cylindrical surface, and $\Phi$ is the angle at $\vec{R}(s)$ between the osculating plane to $\vec{R}$ and the tangent plane to the cylinder, with sign given by the right-hand rule with respect to the direction of increasing $s$. Therefore

$$
\kappa^{2}=\gamma^{2}+k^{2}
$$


and the energy density can be written

$$
\frac{1}{2}\left(a_{1} \gamma(s)^{2}+a_{1} k(s)^{2}+a_{2} \kappa_{2}(s)^{2}+a_{3} \tau(s)^{2}\right)
$$

The integral of this function of $s$ between 0 and $s_{\text {final }}$ is the total strain energy. By Dirichlet's Principle the desired base curve can be obtained by minimizing it as a functional of $\vec{R}$.

If $a_{2}$ and $a_{3}$ were equal to zero, the minimizing curve would instead be the equilibrium configuration of a thin elastic wire of circular cross section, straight in its unstressed state but constrained to lie in the cylindrical surface and subject to torques about axes perpendicular to the surface and at the endpoints of the wire only. If the cylindrical surface were a flat plane then the normal curvature $k$ would be zero and our problem would just be the classical Bernoulli-Euler problem of the "elastica". Then the solution would be contained in standard works on the subject, of which the prize-winning habilitation dissertation of Max Born [2] is one of the best. For us the surface is not flat and $a_{2}$ and $a_{3}$ are not zero but the curvature of the surface is easily taken into account and the last two terms can be regarded as given functions, predetermined in the other step, Step 2 of the overall iterative process. In this step, Step 1, we do not get a closed-form solution in terms of elliptic integrals as in the classical case, but Born's method can be modified to give us a solution by only one numerical quadrature.

The curve will be contained entirely within the top half of the cylindrical surface so first we set up there a conveniently placed two-dimensional coordinate system. Because the surface is developable we can coordinatize it as a $(u, v)$-plane. Let the $u$-axis be perpendicular and the $v$-axis be parallel to the rulings of the cylinder. The direction of increasing $u$ is to be such that the right-hand rule from $u$ to $v$ indicates a normal to the surface oriented so as to point radially away from the axis of the cylinder. The origin of the $(u, v)$-coordinates is to be placed at the beginning of the base curve where it is tangent to the ruling followed by the cable along the straight section of the dipole. As arc length $s$ increases from $s=0$ at its beginning, the base curve proceeds in the direction of increasing $v$, bending over in the direction of decreasing $u$ until at its endpoint, $s=s_{\text {final }}$, it is perpendicular to the ruling along the top of the cylinder at $u=-a$ where $a$ is the half-width of the total $180^{\circ}$-bend made by the cable at the end of the dipole before it starts back down the long straight section. By symmetry we need only find half of that curve.

Let $\psi(s)$ be the angle made with the $u$-axis by the tangent to the base curve at $s$. 
Then

$$
\gamma=\psi^{\prime}
$$

and, from the second fundamental form for the surface (equation (11) on page 290 of [4]) we get

$$
k=k_{c} \cos ^{2} \psi
$$

by means of equation (12) on page 288 of [4], where $k_{c}$ is the reciprocal of the radius of the cylinder.

The last two terms in expression (5) above are presumed given from Step 2. All of the terms except the first can be lumped together into one functional $h$ of $\psi$ (containing derivatives of $\psi$ ). After dividing through by the constant $a_{1}$, the functional of $\vec{R}$ to be minimized is

$$
\int_{0}^{a f i n a l}\left(\frac{1}{2}\left(\psi^{\prime}(s)\right)^{2}+(h(\psi))(s)\right) d s
$$

subject to the initial conditions $u(0)=0, v(0)=0, \psi(0)=\pi / 2$, and the value of $\psi^{\prime}(0)$, which is at our disposal; and also subject to the final conditions $u\left(s_{\text {final }}\right)=-a, v\left(s_{\text {final }}\right)=b$ and $\psi\left(s_{\text {final }}\right)=\pi$.

In $(s, \psi, u, v)$-space we want to join the two points $(0, \pi / 2,0,0)$ and $\left(s_{f i n a l}, \pi,-a, b\right)$ by a minimizing curve, where $a$ and $b$ are input by the user and $s_{\text {final }}$ will be determined by the terminal condition on $\psi$.

Define the Lagrangian function

$$
L=\frac{1}{2}\left(\psi^{\prime}\right)^{2}+h(\psi)+\lambda\left(\cos \psi-u^{\prime}\right)+\mu\left(\sin \psi-v^{\prime}\right)
$$

to replace the minimization problem by a minimax problem. Then the five Euler-Lagrange equations for the five unknown functions $\psi, u, v, \lambda$, and $\mu$, of $s$, are

$$
\begin{aligned}
\psi^{\prime \prime}-h^{\prime}(\psi)+\lambda \sin \psi-\mu \cos \psi & =0, \\
\lambda^{\prime} & =0, \\
\mu^{\prime} & =0, \\
\cos \psi-u^{\prime} & =0, \\
\sin \psi-v^{\prime} & =0,
\end{aligned}
$$


where $h^{\prime}$ is the functional derivative of $h$ (see Ch. IV, $\S 8.1$ of [5]).

The second and third equations show that the Lagrange multipliers $\lambda$ and $\mu$ are constants to be determined by the other conditions to be satisfied. Re-express them in terms of two other constants, $r_{0}$ and $\psi_{0}$, by $\lambda=r_{0} \cos \psi_{0}$ and $\mu=r_{0} \sin \psi_{0}$. Then

$$
\psi^{\prime \prime}-h^{\prime}(\psi)+r_{0} \sin \left(\psi-\psi_{0}\right)=0
$$

By symmetry about the vertical midplane along the axis of the cylinder, $\psi^{\prime \prime}$ and $h^{\prime}(\psi)$ will equal 0 at $s_{\text {final }}$, so $\psi_{0}$ must equal either 0 or $\pi$. This choice only changes the sign of $r_{0}$, so it can be absorbed into that parameter and we can arbitrarily choose $\psi_{0}=\pi$.

To get a first integral of the first of the Euler-Lagrange differential equations, multiply both sides by $\psi^{\prime}$ (as in the classical solution to the equation of motion of a pendulum, page 115 in [7], which is closely related to our own problem):

$$
\psi^{\prime \prime} \psi^{\prime}=h^{\prime}(\psi) \psi^{\prime}+r_{0} \sin (\psi) \psi^{\prime}
$$

Then, by integration,

$$
\frac{1}{2}\left(\psi^{\prime}(s)^{2}-\psi^{\prime}(0)^{2}\right)=\int_{0}^{s} h^{\prime}(\psi) \psi^{\prime} d s-\left[r_{0} \cos (\psi)\right]_{0}^{s}=H(s)-r_{0} \cos (\psi(s))
$$

Therefore $\psi^{\prime}=f(\psi) \stackrel{\text { def }}{=} \sqrt{2\left(H(s)-r_{0} \cos \psi(s)\right)+\psi^{\prime}(0)^{2}}$. Now we can solve for $s$ as a function of $\psi$ by one numerical quadrature:

$$
s=\int_{\pi / 2}^{\psi} \frac{d \psi}{f(\psi)}
$$

where the monotonicity of $\psi$, from Step 2, permits us to invert it, $s=s_{S t e p 2}(\psi)$, and express $H$ as a known function of $\psi$.

To get $u$ and $v$, remember that $d x / d \psi=(d x / d s)(d s / d \psi)=\cos \psi / f(\psi)$, and then integrate the fourth and fifth Euler-Lagrange equations numerically:

$$
u=\int_{\pi / 2}^{\psi} \frac{\cos \psi}{f(\psi)} d \psi
$$

and 


$$
v=\int_{\pi / 2}^{\psi} \frac{\sin \psi}{f(\psi)} d \psi
$$

The $u(\pi)$ and $v(\pi)$ thus obtained are functions of two constants of integration, $r_{0}$ and $\psi^{\prime}(0)$. We want to find the values of these two parameters which make $u(\pi)=-a$ and $v(\pi)=b$. There is a large and sophisticated literature on the solution of $N$ nonlinear equations in $N$ unknowns but in our case $N$ is only equal to 2 so we employ an unsophisticated algorithm, easy to code, guaranteed to work, and fast enough for interactive use: Vary $r_{0}$ by regula falsa (see page 248 in [13]) until $u(\pi)=-a$, and surround this inner loop on $r_{0}$ by an outer loop in which $\psi^{\prime}(0)$ is varied, also by regula falsa, until $v(\pi)=b$.

\section{The Rectifying Developable}

A developable surface is the envelope of a one-parameter family of planes. If the bent rectangular strip is contained in a developable surface then it is the union of a family of straight line segments in one-to-one correspondence with the value of the parameter $s$ for which each segment contains the corresponding point $\overrightarrow{R(s)}$ on the base curve. These segments are the points of intersection of the enveloping planes with the strip. They are the rulings of the developable surface (see page $314, \S 142$ of [4]). If no two of the segments intersect then each point in the strip lies on one and only one ruling. The points are equally distributed along the rulings at the same distances from the base curve that they were distributed from the edge of the planar rectangle along the straight line segments they formed there so, given the base curve, the position of the strip in space will be completely determined once we have found its rulings.

In the case of the rectifying developable the rulings are known (see top of page 47 in [15]) to be parallel to the Darboux vector

$$
\vec{\delta}=\tau \vec{T}+\kappa \vec{B}
$$

(equation (5) on page 93 of [4]). $\tau$ is the torsion and $\kappa$ the curvature of the curve, and $\vec{T}$ and $\vec{B}$ are two of the three mutually orthogonal vectors of unit length making up the moving trihedral associated with any smooth curve in space:

$\vec{T}$ is tangent to the curve in the direction of increasing arc length $s$, 
$\vec{N}$ points in the direction of the principal normal to the curve, and,

$\vec{B}=\vec{T} \times \vec{N}$ is the binormal to the curve.

These vectors are related to each other by the famous Frenet Formulas of classical differential geometry

$$
\begin{aligned}
& \frac{d \vec{T}}{d s}=\kappa \vec{N}, \\
& \frac{d \vec{N}}{d s}=-\kappa \vec{T}+\tau \vec{B}, \\
& \frac{d \vec{B}}{d s}=-\tau \vec{N}
\end{aligned}
$$

(equations (4) on page 93 of [4]), and they can all be constructed from values of variables available during construction of the base curve.

The Frenet vectors form the basis for a family of three-dimensional coordinate systems attached to the cables in a natural and useful way. They also enable us to construct. a twodimensional coordinate system intrinsic in the strip so that the coordinates of points in the strip are unchanged by the bending transformation. Then it is easy to find the point in the planar strip that corresponds to each point in the bent strip. We know not only the configuration of the strip in space but also the length-preserving transformation (isometry) that put it there. Later, when the strip is twisted to match the condition at $s=0$, or otherwise deformed nonisometrically to incorporate empirical information obtained from coil winding experience, we can still keep track of each point and therefore the local strains.

There is an additional need for the Frenet basis in the computer program where more detail is put into the internal geometry of the cables. They are given a trapezoidal cross section, not the zero thickness of the strip, and they are packed together tightly into groups with no interstices inside each group anywhere along the winding. The resulting structure is too intricate to be specified in one fixed coordinate system.

To get $\vec{T}$ in a Cartesian coordinate system $(x, y, z)$ with $z$-axis parallel to the axis of the cylinder, let $\zeta$ be the angle made with the $x$-axis, measured towards the $y$-axis, of the projection of $\vec{R}(s)$ upon the $(x, y)$-plane. Then

$$
\vec{T}=(-\cos \psi \sin \zeta, \cos \psi \cos \zeta, \sin \psi)
$$


To get the principal normal $\vec{N}$, note that the geodesic curvature (see equation (2) above) is $\gamma=d \psi(s) / d s$ where $d s=d \psi / f(\psi)$, so $\gamma=f(\psi)$. The normal curvature (see equation (3)) is $k=+\vec{T} \cdot d \vec{n} / d s$ by (8) on page 284 of [4] where $\vec{n}$ is the unit normal to the cylinder pointing away from its axis.The + -sign occurs here because $\vec{n}$ is on the opposite side of the surface from $\vec{N}$. The second fundamental form (equation (11) on page 290 of [4]) is simplified for a cylinder,

$$
\frac{d \vec{n}}{d s}=\cos \psi \frac{d \vec{n}}{d u}+\sin \psi \frac{d \vec{n}}{d v}=\cos \psi \frac{d \vec{n}}{d u},
$$

because $d \vec{n} / d v$, the derivative along a ruling of the cylinder, is equal to $\overrightarrow{0}$. Therefore

$$
\frac{d \vec{n}}{d s}=k_{c} \cos \psi \frac{d \vec{n}}{d \zeta}
$$

and, as before, $k=k_{c} \cos ^{2} \psi$, where $1 / k_{c}$ is the radius of the cylinder. So we know the curvature $\kappa=\sqrt{\gamma^{2}+k^{2}}$ by equations (2) and (3). Therefore we know the angle $\phi$ between $\vec{N}$ and $\vec{n}$ (see figure 130 on page 283 of [4]) because $\pi-\phi=\arcsin (\gamma / \kappa)$ by equation (2) where we have chosen that branch of arcsin corresponding again to the fact that $\vec{n}$ and $\vec{N}$ are on opposite sides of the surface. So we know $\vec{N}$.

We can get $\vec{B}$ directly from its definition, $\vec{B}=\vec{T} \times \vec{N}$.

Finally, to get $\vec{\delta}=\tau \vec{T}+\kappa \vec{B}$ we need only find $\tau$. But by equation (4) on page 284 of [4], $\tau=t-\phi^{\prime}$, where the geodesic torsion $t=-(\vec{n} \times \vec{T}) \cdot d \vec{n} / d s$ by equation (8) on that page. So $t=-k_{c} \cos \psi \cos (\psi+\pi / 2)=k_{c} \cos \psi \sin \psi$. To finish the evaluation of $\tau$ we find $\phi^{\prime}$ by performing the differentiation

$$
\begin{aligned}
\phi^{\prime} & =-\frac{d}{d s}(\pi-\phi) \\
& =-\frac{d}{d s} \arcsin \frac{\gamma}{\kappa} \\
& =\frac{k^{\prime} \gamma-k \gamma^{\prime}}{\gamma^{2}+k^{2}},
\end{aligned}
$$

where

$$
\gamma^{\prime}=\psi^{\prime \prime}=h^{\prime}(\psi)+r_{0} \sin \psi
$$


and

$$
k^{\prime}=-2 k_{c} \gamma \cos \psi \sin \psi
$$

So the values of all the variables determining $\phi^{\prime}$, and hence $\tau$, and hence the Darboux vector and the developable strip, are known.

\section{Twisting the Rectifying Developable}

The base curve determines the rectifying developable. Hence it determines the inclination of the strip at $s=0$ where the conductor leaves the long straight section of the dipole and enters the bend at its end. But along the straight section the strip is modeled by a planar rectangle whose inclination may be different from that determined by the base curve. It may be necessary to twist the developable strip in order to make a smooth transition with the straight section even though developability will then be lost. Early experience encourages us to believe that for SSC dipole magnets this can be done with an acceptably small increase in strain energy.

To show that the rectifying developable at $s=0$ must be perpendicular to the surface of the cylinder, we show that the osculating plane of the base curve must be tangent to the cylinder there. By equation (3) above it will be sufficient to prove that $k(s) / \kappa(s) \rightarrow 0$ as $s \downarrow 0$. Therefore by equations (6), (7), and (4) it will be sufficient to prove that $\cos ^{2} \psi / \sqrt{\psi^{\prime 2}+k^{2}} \rightarrow 0$. But this expression is dominated by $\sin ^{2} \epsilon / \epsilon^{\prime}$ where $\epsilon(s)=\psi(s)-\pi / 2$. We keep $\epsilon$ smooth so that it has an expansion of the form $\epsilon(s)=c_{n} s^{n}+c_{n+1} s^{n+1}+\ldots$ where, by the initial condition $\psi(0)=\pi / 2$ on $\psi, n>0$. Therefore the numerator of the dominating expression is of order $2 n$ in $s$ whereas its denominator is only of order $n-1$, so it must go to zero, completing the proof that the rectifying developable is perpendicular to the cylinder at $s=0$.

In general $\psi^{\prime}(0) \neq 0$, so $\kappa(0) \neq 0$ and, because $\tau(0)=0$ by continuity from $\tau(s)=0$ for $s<0$ along the straight section, the ruling of the rectifying developable will, by equation (8), exist and be perpendicular to the cylinder at $s=0$. By symmetry, $\tau\left(s_{\text {final }}\right)=0$, so the ruling will be perpendicular to the base curve at $s=s_{\text {final }}$. In the twisting operation, the first ruling is rotated about the base curve until the twisted strip joins smoothly with the strip as given along the straight section of the dipole. The twist is distributed smoothly along the interval $0 \leq s \leq s_{\text {final }}$ until it reaches the second ruling, which is left fixed, although the 
computer program also provides an opportunity to twist the second ruling, for example to avoid excessive curvature of the free edge of the strip at the nose of the winding.

The angle of twist of the strip away from the rectifying developable is the $\varphi(s)$ defined in Section 2 above. We now define its sign to be positive if the direction of rotation through an acute angle from $N \overrightarrow{(s)}$ to the vector radius of curvature corresponding to $\kappa_{1}(s)$ is righthanded about the tangent to $\vec{R}$ at $s$. Otherwise take the sign of $\varphi(s)$ to be negative.

The Frenet vector $\vec{N}(s)$ is perpendicular to the rectifying developable at $\vec{R}(s) . \vec{T}(s)$ and $\vec{B}(s)$ are tangent to it there. If we rotate this triple of vectors about $\vec{T}(s)$,

$$
\begin{aligned}
\vec{T}^{(\varphi)}(s) & =\vec{T}(s) \\
\vec{N}^{(\varphi)}(s) & =+\cos \varphi(s) \vec{N}(s)+\sin \varphi(s) \vec{B}(s) \\
\vec{B}^{(\varphi)}(s) & =-\sin \varphi(s) \vec{N}(s)+\cos \varphi(s) \vec{B}(s)
\end{aligned}
$$

then we get a new triple of vectors such that $\vec{N}^{(\varphi)}(s)$ is perpendicular to, and $\vec{T}^{(\varphi)}(s)$ and $\vec{B}^{(\varphi)}(s)$ are tangent to, the twisted strip at $\vec{R}(s)$.

The strip is twisted by rotating its rulings about $\vec{T}$. The point $\lambda \vec{\delta}(s)+\vec{R}(s)$ on the ruling through $\vec{R}(s)$ is rotated to the point $\lambda \vec{\delta}(\varphi)(s)+\vec{R}(s)$ on the corresponding ruling of the twisted strip where $\vec{\delta}(s)$ is a Darboux vector $(8)$ and $\vec{\delta}^{(\varphi)}(s)=\tau(s) \vec{T}(s)+\kappa(s) \vec{B}^{(\varphi)}(s)$.

From the Frenet Formulas,

$$
\begin{aligned}
\kappa_{1}^{(\varphi)}(s) & =\frac{d \vec{T}^{(\varphi)}(s)}{d s} \cdot \vec{N}^{(\varphi)}(s) \\
& =\kappa(s) \vec{N}(s) \cdot \vec{N}^{(\varphi)}(s) \\
& =\kappa(s) \cos \varphi(s) \\
\kappa_{2}^{(\varphi)}(s) & =\left|\frac{d B_{(\varphi)}^{(s)}}{d s} \cdot T^{(\varphi)}(s)\right| \\
& =|\sin \varphi(s) \kappa(s)| \\
\tau^{(\varphi)}(s) & =-\frac{d B^{(\varphi)}(s)}{d s} \cdot \vec{N}^{(\varphi)}(s) \\
& =\tau(s)+\varphi^{\prime}(s) .
\end{aligned}
$$


For Step 2, insert these values into expression (1) and minimize the integral as in Step 1 , but this time as a functional of $\varphi$ with $\vec{R}$ fixed.

The science of numerical optimization has reached a level of development where it can do the minimization we need. The most reliable algorithms are those for unconstrained independent variables. Fortunately our independent variables are unconstrained. QuasiNewton methods (see $\S 10.7$ of [13]) have a good reputation in this domain. They possess good convergence properties in general and in our case have the particular advantage that upon each entry into Step 2 the approximation to the inverse Hessian can be restarted at the point where it was left upon the last exit from Step 2. To the extent that the functional we have chosen to minimize does represent the physical problem, a quasi-Newton method can solve the problem.

\section{Application}

The method described above has been implemented in the computer program BEND, currently being used by a design team headed by Rodger Bossert at Fermi National Laboratory. Some applications were discussed at the Breckenridge Workshop, August 14-24, 1989 $[3]$.

BEND is written in strictly standard ANSI FORTRAN 77, liberally supplied with comment cards containing very detailed references to the mathematical literature. It interfaces with Structural Dynamics Research Corporation (SDRC) drafting system I-DEAS, and the Manufacturing and Consulting Services (MCS) numerically-controlled machining system ANVIL-5000, by means of files designed by Greg Lee. His program AutoEnd [9] integrates the software together into one system. In I-DEAS the files are read by a program written in the I-DEAS Language [6] by Leo A. Thiel of SDRC. In ANVIL the files are read by a program written in FORTRAN and the ANVIL language GRAPL [1] by Bob Andree.

Superconducting cables have complicated elastic properties about which much is known but much more is not. Therefore the loop from Step 2 back to Step 1 at present contains a knowledgeable human operator-knowledgeable in the art of cable winding though not necessarily in the mechanics of running the program. The prompts have proven to be largely self-explanatory. BEND needs interactive input based on cable-winding expertise. The theory with which its design was started has served primarily as a strong framework for 
the support of pragmatic adaptations to reality.

The classical, quadratic, total strain energy given by expression (1) above is based on a simple linear Hooke's Law. It can be improved upon as a figure of merit. More appropriate than a quadratic norm is a $p$-norm, one in which the $p^{\text {th }}$ root of the weighted integral of the $p=2$ power of the flexure and torsion is replaced by an integral with $p>>2$. Then it is an approximation to the supremum norm $p=\infty$ which expresses on the one hand our lack of concern about the strain of the cable below its damage threshold and, on the other, our primary concern that the threshold not be exceeded. A value $p<\infty$ is preferred because then the norm is a differentiable function of the cable configuration for minimization. It is not differentiable for $p=\infty$.

The cable has a built-in tendency to twist about $\vec{T}$ more readily in one direction than another, inducing a slight asymmetry about the vertical midplane of a dipole that may be noticeable at the nose of the coil. A norm can not conveniently express this tendency. Instead the asymmetry is put into the strip by an ad hoc, slightly skewed, affine transformation of the data just before output from BEND.

The stress-strain relations should be anisotropic and nonlinear but we do not have the experimental data. However we do have good estimates of the flexure and torsion limits beyond which there is danger of damage, so that is the criterion monitored most closely.

The curvature of the free edge of the strip at the nose of the winding sometimes becomes dangerously large. The rulings of the rectifying developable may tend towards a common focus there. The Euler-Kirchhoff model assumes that the width of the strip is too small for this to happen. When it does, the program provides for another ad hoc remedy in which the curvature is decreased while attempting to minimize the accompanying increase in deviation from developability.

The most serious complication is caused by the fact that the cable is not a strip of zero thickness. Its transverse cross section is not even rectangular. It is slightly trapezoidal ("keystoning") with slightly rounded corners. These shapes must be held precisely against each other and against the cylindrical bore in groups of up to ten or fifteen cables. As they twist their way around the end of the magnet, the relative positions of the cable cross sections shift within the group cross section, causing the latter to vary. This varying cross section is determined by a lengthy program segment full of high school trigonometry and undergraduate vector analysis. In the process there occurs a deterioration of our attempt to 
be mathematically precise about minimizing strain. The zero-thickness strip produced by the mathematical theory is used only as a guide for the group of positive-thickness cables. The strip is placed between two cables somewhere in the group, interactively by the operator. The rest of the cables are packed alongside. The farther they are from the guiding strip the farther they are outside the domain of relevance of the analysis that optimized the strip. Selected information about strains in all of the cables is presented to the operator to provide assistance in the trial-and-error process of optimal placement of the strip in the group.

The original assumption of small $\varphi$ has been violated in some applications but the resulting cable configuration has looked reasonable. Damaging flexure and torsion was avoided but we do not know how close the strain energy was to its minimum.

The separation of the iteration into two steps, on two disjoint subsets of the independent variables, has an advantage of allowing a natural insertion point for expertise that has not yet been formalized. We still expect to automate the procedure after more experience has been gained.

Fifty points along the base curve were quite enough to give an accurate numerical solution of the Euler-Lagrange equation in Step 1 and a smooth curve for the CADCAM programs to work on. The equal spacing in $\psi$, rather than in arc length $s$, gave a better approximation in regions of large curvature where it is needed.

Numerical integration of the equation with fifty points takes a negligible amount of CPU time on a VAX 8650. Quasi-Newton with fifty points in Step 2 is feasible but the computation time is not negligible so $\varphi$ has been reparameterized to depend on only six parameters, physically meaningful for interactive use. The linear term in a perturbation expansion about $\varphi=0$ of the solution of the nonlinear equations for the twist of a planar strip, is a cubic spline. We assume that the solution to our problem can be represented with sufficient accuracy by the six-parameter family of deformations of such a spline.

Financial and professional support for this work has come from Fermilab where $I$ have been on loan from Argonne National Laboratory. I depend on Bossert's group for information about the mechanics of real coil winding. I am also indebted to the management of my home project, the Advanced Photon Source at Argonne, for a far-seeing attitude towards research and development not directly relevant to their immediate needs. 


\section{References}

[1] ANVIL-5000, GRAPL, Manufacturing and Consulting Services, Inc., 6 Hughes, Irvine, California 92718.

[2] M. Born, Stabilität der elastischen Linie in Ebene und Raum, reprinted as Chapter I in his Ausgewählte Abhandlungen, vol. I, Vandenhoeck \& Ruprecht, Göttingen (1963).

[3] R. C. Bossert, J. M. Cook and J. S. Brandt, End Designs for Superconducting Magnets, presented at Breckenridge Workshop, August 14-24, 1989.

[4] Louis Brand, Vector and Tensor Analysis, John Wiley \& Sons, Inc., New York (1947).

[5] R. Courant and D. Hilbert, Methods of Mathematical Physics, Vol. I, Interscience Publishers, Inc., New York (1953).

[6] I-DEAS User's Guide, SDRC World Headquaters, 2000 Eastman Drive, Milfor, Ohio 45150.

[7] T. v. Kármán and M. A. Biot, Mathematical Methods in Engineering, McGraw-Hill Book Co., New York (1940).

[8] L. Lederman, The Supercollider: Assault on the Summit, in Modern Physics in America, W. Fickinger and K. Kowalski, Editors, AIP Conference Proceedings 169, American Institute of Physics, New York (1988).

[9] Gregory C. Lee, AutoEnd, Version 1.0, Fermi National Accelerator Laboratory, Technical Support Section, Batavia, IL (August 12, 1989).

[10] A. E. H. Love, A Treatise on the Mathematical Theory of Elasticity, Macmillan Co., New York (1944).

[11] J. C. Maxwell, The Scientific Papers of James Clerk Maxwell, vol. I, Dover Publications, Inc., New York (1965).

[12] A. V. Pogorelov, Bending of Surfaces and Stability of Shells, American Mathematical Society, Providence (1988). 
[13] W. H. Press, B. P. Flannery, S. A. Teukolsky and W. T. Vtterling, Numerical Recipes, Cambridge University Press, New York (1986).

[14] H. I. Rosten, The Constant Perimeter End, RL-73-096, Applied Physics Division, Rutherford Laboratory, Chilton Berks (September, 1973).

[15] C. E. Weatherburn, Differential Geometry of Three Dimensions, Cambridge University Press, Cambridge (1955). 